\title{
Publisher Correction: Low level equilibrium trap, unemployment, efficiency of education system, child labour and human capital formation
}

\section{Kamalika Chakraborty $^{1}$ (D) Bidisha Chakraborty ${ }^{1}$}

\section{Correction to: J Econ https://doi.org/10.1007/s00712-017-0585-x}

In the original version of the article, equation 4 and the first equation in the Appendix section have been incorrectly published. The correct equations are given below:

$$
\begin{aligned}
\mathrm{U}_{\mathrm{t}} & =\beta_{1} \ln \left(\mathrm{c}_{\mathrm{t}}-\underline{\mathrm{c}}\right)+\beta_{2} \ln \left[\mathrm{f} \delta\left(\mathrm{bs}_{\mathrm{t}} \mathrm{h}_{\mathrm{t}}+\underline{\mathrm{h}}\right)+(1-\mathrm{f}) \mathrm{A}\right] \quad \text { if } \mathrm{c}_{\mathrm{t}} \geq \underline{\mathrm{c}} \\
& =-\infty \text { otherwise; }
\end{aligned}
$$

$$
\begin{aligned}
\mathrm{Z}= & \beta_{1} \ln \left(\mathrm{c}_{\mathrm{t}}-\underline{\mathrm{c}}\right)+\beta_{2} \ln \left[\mathrm{f} \delta\left(\mathrm{bs}_{\mathrm{t}} \mathrm{h}_{\mathrm{t}}+\underline{\mathrm{h}}\right)+(1-\mathrm{f}) \mathrm{A}\right]+\lambda\left[\mathrm{A}+\mathrm{A} \varphi\left(1-\mathrm{s}_{\mathrm{t}}\right)\right. \\
& \left.-\mathrm{p}_{\mathrm{c}} \mathrm{c}_{\mathrm{t}}\right]+\theta\left(\mathrm{c}_{\mathrm{t}}-\underline{\mathrm{c}}\right),
\end{aligned}
$$

where $\lambda$ is the Lagrange multiplier.

The original article can be found online at https://doi.org/10.1007/s00712-017-0585-x.

Kamalika Chakraborty

k.chakraborty82@gmail.com

1 Jadavpur University, 188 Raja S.C.Mallick Road, Kolkata, West Bengal 700032, India 